\title{
Fetal Neurodevelopmental Disorder
}

National Cancer Institute

\section{Source}

National Cancer Institute. Fetal Neurodevelopmental Disorder. NCI Thesaurus. Code

C103236.

A fetal affliction that has a neurological basis and manifests as a developmental disability. 